\title{
Load-shifting brace treatment for osteoarthritis of the knee: A minimum 2 1/2-year follow-up study
}

\author{
Nicholas J. Giori, MD, PhD \\ Surgical Service and Rehabilitation Research and Development Center, Department of Veterans Affairs (VA) Palo Alto \\ Health Care System, Palo Alto, CA; Department of Orthopedic Surgery and Sports Medicine, Stanford University, \\ Stanford, $C A$
}

\begin{abstract}
Objectives in treating primarily unicompartmental knee arthritis with a load-shifting brace are pain relief, compliance, brace durability, and complication-free treatment over multiple years. This was a single institution retrospective chart review, radiograph review, and telephone survey of patients treated from 1997 to 1999 with a load-shifting knee brace. Forty-six patients (49 knees) with a minimum 2 1/2-year follow-up (average 3.3 years) were reviewed. Kaplan-Meier survivorship analysis revealed that load-shifting brace use had a survival of $76 \%$ at 1 year, $69 \%$ at 2 years, and $61 \%$ at 3 years. Younger patients had a higher likelihood of longer brace use than older patients. One patient had ipsilateral leg swelling and a pulmonary embolus after initiating bracing. Eliminating the high numbers of early failures would be desirable. One should be aware of the potential complication of venous thrombosis and thromboembolism.
\end{abstract}

Key words: brace, knee, osteoarthritis, survivorship.

\section{INTRODUCTION}

Osteoarthritis of the knee involving primarily one compartment is commonly encountered in the orthopedic clinic. Treatment options include conservative management with medications, bracing, osteotomy, unicompartmental arthroplasty, and total knee arthroplasty. Conservative management with medications or strengthening is commonly done for mildly affected patients, while severely affected patients in an older age group who are less physically active can be effectively treated with unicompartmental arthroplasty or total knee arthroplasty. Younger, active patients who do not respond to conservative measures, or patients with other medical problems that prohibit surgery, may be treated in a loadshifting brace.

A load-shifting brace for osteoarthritis involving primarily one compartment of the knee incorporates a rigid frame, a hinge mechanism, and straps to apply a bending moment to the knee. The brace applies either a valgus or varus moment to the knee, unloading either the medial or lateral compartment. It is custom-fitted to the patient and can cost from US $\$ 1,500$ to $\$ 2,000$.

Studies of patients treated with a load-shifting brace have demonstrated pain relief, functional improvement, gait improvement, improvement in knee scores, and improvement in the adduction moment across the knee [1-3]. Fluoroscopic analysis of these patients during walking has also revealed separation of the condyles on the unloaded side during heal-strike phase of gait [4].

Abbreviations: $\mathrm{BMI}=$ body mass index, $\mathrm{CI}=$ confidence interval, SD = standard deviation.

The work published in this manuscript was unfunded at the time of manuscript preparation.

Address all correspondence to Nicholas J. Giori, MD, PhD; Surgical Service (112), VA Palo Alto Health Care System, Palo Alto, CA 94304; 650-493-5000, ext. 64765; fax: 650-8523430; email: ngiori@stanford.edu. 
These studies, however, investigated only immediate changes with bracing, or changes with follow-up of up to 6 months. Two studies of bracing for osteoarthritis of the knee on a total of 33 patients reported pain relief, improved knee scores, and improved quadriceps strength at 1 year, and one recent study on 30 patients found pain relief, decreased use of pain medication, and functional improvement at 2 years [5-7].

Bracing is often employed as a treatment strategy for knee arthritis involving primarily one compartment to relieve pain, to maintain function and activity level, and in the case of the younger, active patient, to delay an eventual knee arthroplasty procedure. Primary treatment objectives, then, are sufficient pain relief, compliance, brace durability, and complication-free treatment over a multiple-year time period. At present, no published information exists on the success of load-shifting brace use in meeting these objectives. The high cost of the brace and concern about early failures limit enthusiasm for this treatment strategy. This study describes the minimum 2 1/2-year results of bracing for osteoarthritis of the knee involving primarily one compartment at one institution and makes recommendations for improving outcomes.

\section{MATERIALS AND METHODS}

This was a retrospective review with approval obtained from the institutional review board. A list of all load-shifting braces provided by the prosthetics department at this institution for a diagnosis of osteoarthritis from 1997 to 1999 was generated. The patients' date of birth, sex, height, weight, and subsequent operative interventions were drawn from the computerized medical record. The date each brace was issued was drawn from the prosthetist's records.

Each patient was then contacted by telephone and responded to a telephone survey. Patients were asked whether or not they were still using the brace. If not, they were asked when they stopped using the brace and why. If they were still using the brace, they were asked when and how often they used the brace. A patient was considered to be using the brace if he or she was regularly and predictably using the brace at least once a week. Usually, this constituted using the brace for a regularly occurring activity such as work, exercise, or shopping. For this analysis, patients who used the brace less than once a week were considered to not be using the brace. Those patients were then asked when they stopped using the brace once a week; this was considered the end point of their brace use.

All patients were also asked if they had fit, skin reaction, mechanical breakage, or other problems with the brace. Complications of brace use were also noted. They were then asked for a detailed surgical history of the knee that was braced.

To assess the current effectiveness of the brace in relieving pain and improving function, we then asked each patient who was still using the brace standard questions from the Knee Society clinical rating system [8]. Knee Society pain and function scores were then calculated for each patient with and without the use of the brace. Finally, each patient was asked to rate his or her satisfaction with the brace as very satisfied, satisfied, unsatisfied, or very unsatisfied.

Knee radiographs taken when the brace was issued were also reviewed. For each knee, severity of osteoarthritis involvement in each compartment was rated based on the Kellgran-Lawrence rating system [9].

\section{STATISTICAL METHODS}

A Kaplan-Meier survivorship analysis was completed to determine the probability of continued brace use as a function of time since brace issue. The end of brace use for any reason, including surgery or dissatisfaction with the brace, was used as the failure end point for this analysis. In the Kaplan-Meier analysis, patients lost to follow-up have no impact on the results of the survivorship analysis because they can be considered to have been censored immediately. To find factors predictive of success or failure of brace use, Cox proportional hazards model was used to examine the effects of patient age, body mass index (BMI), weight, and radiographic osteoarthritis grade on the survival of brace use. The effects of the radiographic osteoarthritis grade in the primarily involved compartment, the less involved compartment, and the difference in radiographic osteoarthritis grade between the more and less involved compartments were evaluated. No prior power or sample-size calculation was performed, because the study attempted to follow the results of all braces issued. The 95 percent confidence intervals (CIs) for the hazard ratios in the Cox model provide a measure of the "posterior precision" or statistical power actually achieved by the sample. In the 
three patients with both knees braced, the possibility that knee survival is correlated within individuals was ignored. The result may be a slight underestimate of the standard errors and $p$-values.

\section{RESULTS}

From 1997 to 1999, 55 patients were given a customfitted load-shifting brace for osteoarthritis involving primarily one compartment of the knee. No off-the-shelf braces were included in this study. Seven were lost to follow-up, one died, and one declined participation in the study, leaving forty-six patients. In this group, 23 braces were given for the right leg only, 20 were for the left leg only, and 3 sets (6 braces) were for both legs. Thus, 49 braced legs were analyzed in this study. Results are summarized in Table 1. Thirty-three braces were the DonJoy Defiance Brace (dj Orthopedics Inc., Vista, California, USA), nine braces were the DonJoy Monarch Brace, and seven were the MOS Genu Brace (Bauerfeind Orthopedics, Kempen, Germany). Braces were given for medial compartment arthritis in 43 knees and for lateral compartment arthritis in 6 knees.

This study's participants included 45 men and 1 woman. The average age of the patients when the brace was issued was 57 years (standard deviation [SD] = 12 years).
Average height was $178 \mathrm{~cm}(\mathrm{SD}=8 \mathrm{~cm})$, and average weight was $101 \mathrm{~kg}$ (SD = $20 \mathrm{~kg}$ ). The average BMI was thus $32 \mathrm{~kg} / \mathrm{m}^{2}\left(\mathrm{SD}=6 \mathrm{~kg} / \mathrm{m}^{2}\right)$. Twenty-seven patients (30 knees) met criteria for obesity, with a BMI of $30 \mathrm{~kg} / \mathrm{m}^{2}$ or more.

Minimum follow-up time from the date the brace was issued was 2.5 years. Average follow-up was 3.3 years. The Kaplan-Meier survivorship curve (with 95\% CIs) for these 49 braced knees is shown in the Figure.

Brace use was discontinued within 6 months in 10 of 49 knees (20\%). Seven did not experience relief or felt that the brace was too awkward to use, one changed his medications and felt he did not need the brace, and two experienced unilateral leg swelling. One of these two patients developed dyspnea and was found to have a pulmonary embolus. All of these patients either reported that they never had relief, were dissatisfied with the brace, or had the complications just noted within the first month of brace use.

Brace use was discontinued beyond 6 months in 14 knees. In six knees, the patients reported that the knee felt better and the brace was no longer needed. One patient reported that he stopped using it because of inconvenience, even though it helped. Seven knees underwent total knee arthroplasty at an average of 2.5 years after the brace was issued. These patients underwent total knee arthroplasty for inadequate pain relief with bracing.

Table 1.

Data summary.

\begin{tabular}{|c|c|c|c|c|c|c|c|c|}
\hline Knee & $\begin{array}{c}\text { Height } \\
\text { (cm) }\end{array}$ & $\begin{array}{c}\text { Weight } \\
\text { (kg) }\end{array}$ & $\begin{array}{c}\text { BMI } \\
\left(\mathrm{kg} / \mathrm{m}^{2}\right)\end{array}$ & $\begin{array}{c}\text { Age } \\
\text { When Braced } \\
\text { (Days) }\end{array}$ & $\begin{array}{c}\text { Primary } \\
\text { Compartment } \\
(\mathrm{m} \text { or } \mathrm{l})^{*}\end{array}$ & $\begin{array}{c}\text { OA Grade } \\
\text { Primary Side }\end{array}$ & $\begin{array}{l}\text { End Point } \\
\text { (Days) }\end{array}$ & $\begin{array}{l}\text { Censored (C) } \\
\text { or Failed (F) }\end{array}$ \\
\hline 2 & 180 & 94 & 29.0 & 16,541 & $\mathrm{~m}$ & 1 & 30 & $\mathrm{~F}$ \\
\hline 3 & 173 & 86 & 28.9 & 14,414 & $\mathrm{~m}$ & 2 & 1,093 & $\mathrm{C}$ \\
\hline 6 & 168 & 90 & 32.0 & 26,534 & $\mathrm{~m}$ & 3 & 1,118 & $\mathrm{C}$ \\
\hline 7 & 170 & 84 & 29.0 & 21,460 & $\mathrm{~m}$ & 3 & 889 & $\mathrm{~F}$ \\
\hline 8 & 183 & 111 & 33.2 & 18,871 & $\mathrm{~m}$ & 4 & 1,124 & $\mathrm{C}$ \\
\hline 9 & 188 & 87 & 24.6 & 26,777 & $\mathrm{~m}$ & 3 & 1,331 & $\mathrm{C}$ \\
\hline 13 & 185 & 113 & 32.8 & 15,302 & $\mathrm{~m}$ & 2 & 1,332 & $\mathrm{C}$ \\
\hline
\end{tabular}


JRRD, Volume 41, Number 2, 2004

Table 1. (Continued)

Data summary.

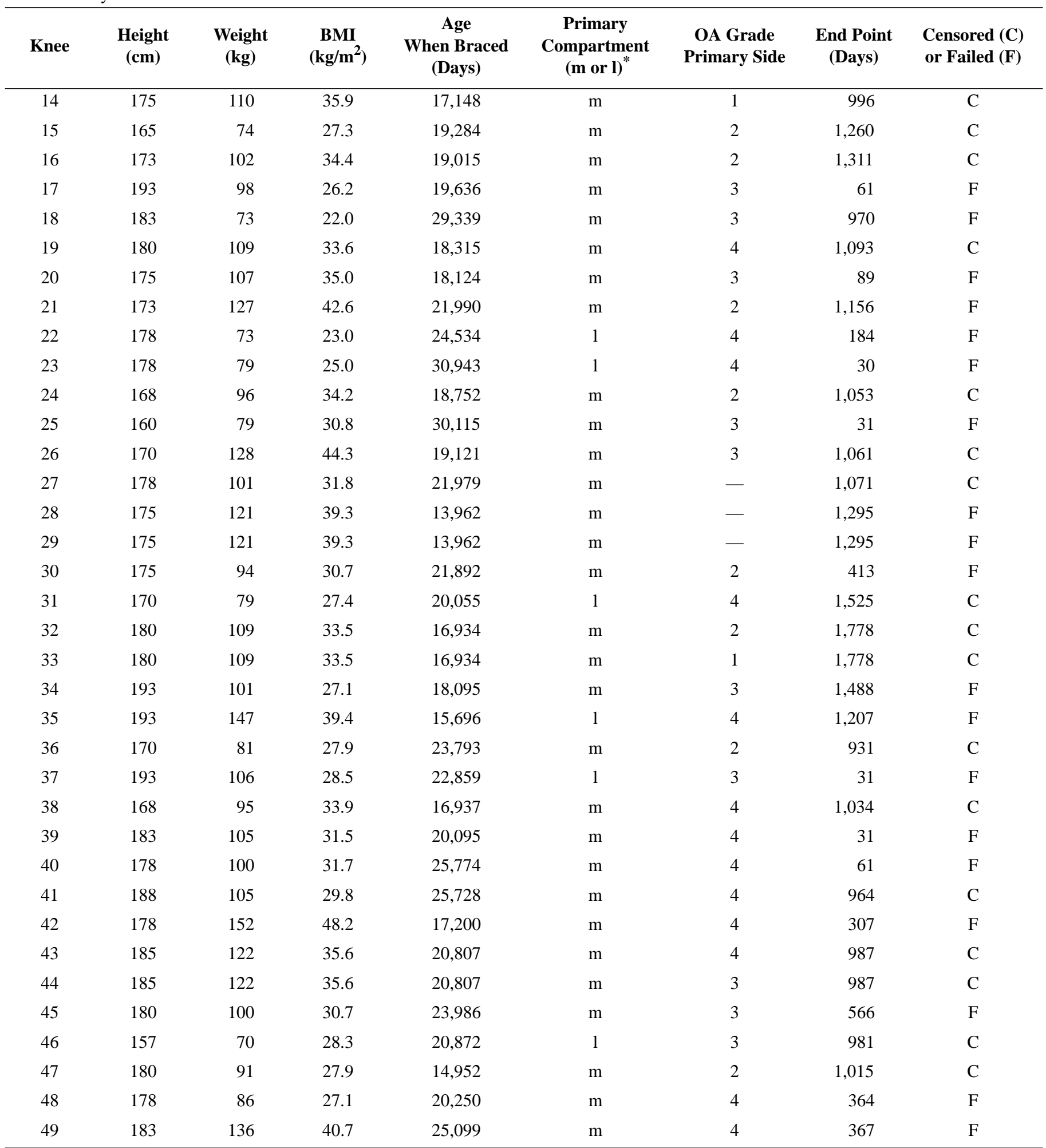

${ }^{*} \mathrm{~m}$ = medial compartment and $\mathrm{l}=$ lateral compartment 


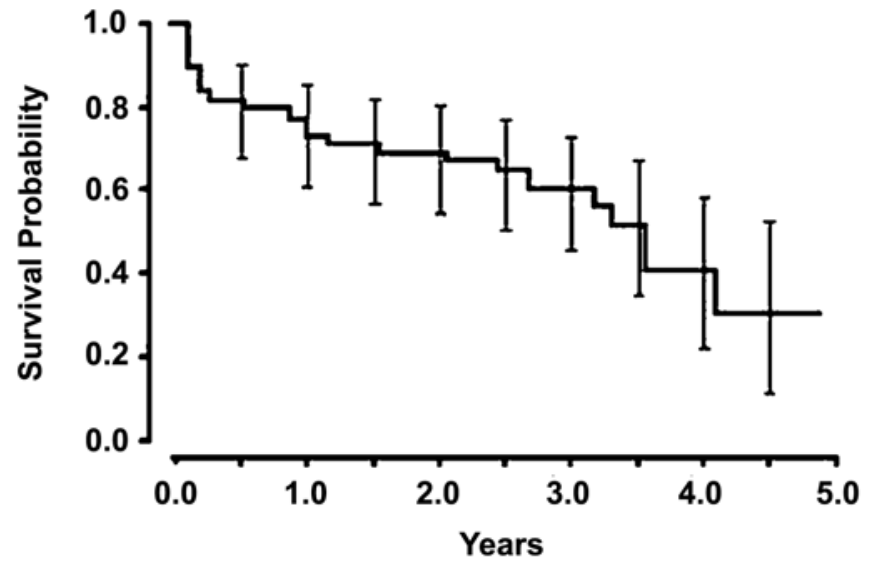

Figure.

Kaplan-Meier survivorship curve with 95\% CIs for load-shifting brace use of all knees. End of brace use for any reason, including surgery or dissatisfaction with the brace, was used as failure end point for this analysis.

Among the 22 patients (25 knees) who were found still to be using the brace when they were contacted, only 6 reported using the brace full time. The remaining 16 patients reported using the brace only as needed, particularly when they were being active or at work. In patients still using the brace, Knee Society pain scores improved an average of 26 points with use of the brace, from 13 to 39 points. Knee Society function scores did rise, but not dramatically, from an average of 61 points to
72 points. Among these 22 patients ( 25 knees) who were still using the brace when they were contacted, 6 reported problems with skin irritation, 2 reported some mechanical problem with the brace, 1 reported a problem with the fit of the brace, and 12 reported no problems with the brace. Four knees, two that were braced for 4 years and two that were braced for 5 years, wore out one brace and were issued a second.

Radiographs taken at the time of initial bracing were available for review in 46 of the 49 knees. In the more involved compartment, 3 knees had grade 1 osteoarthritis, 12 knees had grade 2, 15 knees had grade 3, and 16 knees had grade 4 . In the less-involved compartment, 7 knees had no radiographic evidence of osteoarthritis, 15 knees had grade 1, 12 had grade 2, and 12 had grade 3 osteoarthritis. The difference in grade from the more- to the lessinvolved compartment was 0 in 5 knees, 1 in 25 knees, 2 in 12 knees, and 3 in 4 knees.

Of all the variables considered, only age was found to correlate with length of brace use, with older patients having a higher rate of brace failure than younger patients. The hazard ratio was 1.8 (95\% CIs 1.05 to 3.14, $p=0.032$ by Cox regression) comparing patients at the upper and lower quartile of age (65 and 50 years, respectively). Table 2 lists estimated effect sizes and 95\% CIs for the other variables tested and not found to be statistically significant predictors (BMI, weight, medial or lateral involvement, radiographic osteoarthritis grade in

Table 2.

Table of estimated hazard ratios with 95\% confidence intervals (CIs).

\begin{tabular}{lcccccc}
\hline \multicolumn{1}{c}{ Factor } & Low & High & Difference & Hazard Ratio & Lower 0.95 & Upper 0.95 \\
\hline Age (yr) & 49.5 & 65.1 & 15.6 & 1.82 & 1.05 & 3.14 \\
Body Mass Index & 27.8 & 34.8 & 6.9 & 1.20 & 0.67 & 2.14 \\
OA Primary Side & 2.0 & 4.0 & 2.0 & 2.33 & 0.79 & 6.87 \\
OA Secondary Side & 1.0 & 2.75 & 1.75 & 1.50 & 0.66 & 3.42 \\
OA Difference & 1.0 & 2.0 & 1.0 & 1.15 & 1.38 & 0.67 \\
Weight (lb) & 190.0 & 245.0 & 55.0 & 1.76 & 0.78 & 0.59 \\
Primary Compartment (l:m) & - & - & - & 5.28 \\
\hline
\end{tabular}

Notes:

1. Estimates and CIs for all factors after age are from Cox proportional hazards model, with age included. All factors other than age have 95\% CI, including null value (1.0), and therefore are not significant at $5 \%$ level. However, width of CI shows that for some factors, such as osteoarthritis (OA) primary side, quite high effects cannot be definitively ruled out because of the small number of failures observed.

2. Continuous factors: Hazard ratio estimate and CI compare higher to lower quartile; e.g., effect of age is that a person of age 65.18 (75th percentile) has 1.82 times the hazard of knee failure as a person of age 49.58 (25th percentile). The 95\% CI for this ratio of hazards is from 1.05 to 3.14 , excluding null value (1.0). The estimated hazard ratio would be the same for any two ages separated by same difference in age (15.6 years), by virtue of proportional hazards assumption in Cox model.

3. Primary compartment is a dichotomy; estimate and CI compare l:m (lateral to medial), where "l” is coded as 2 and " $\mathrm{m}$ " as 1. 
each compartment, and difference in radiographic grade between the compartments). Among the 46 patients surveyed, 17 reported being very satisfied with the brace, 19 reported being satisfied, 4 were unsatisfied, and 6 were very unsatisfied with the brace.

\section{DISCUSSION}

A majority of patients who were issued a load-shifting brace for osteoarthritis involving primarily one compartment of the knee were found still to be using their brace and deriving some benefit of pain relief and functional improvement 3 years later. Load-shifting brace use was found to have a survival of 76 percent at 1 year, 69 percent at 2 years, and 61 percent at 3 years after delivery of the brace. Survival of brace use was found to be associated only with age of the patient, younger patients having a higher likelihood of longer brace use than older patients. Longer use of the brace in younger patients may reflect a bias against surgical intervention in this age group.

Other factors, such as radiographic severity of osteoarthritis did not reach statistical significance in the Cox proportional hazards model, but this may be due to the small number of failures observed. One cannot rule out the possibility, based on this study, that other factors may also influence brace use survival.

Interestingly, the highest rate of brace failure occurred within 6 months of brace delivery. About half of the people who had reported discontinuation of brace use reported being dissatisfied with the brace within 1 month of brace delivery. Based on this finding, developing reusable "trial braces" that can easily be sized and fitted to a patient may be cost-effective. Only fitting a permanent brace to those patients who are satisfied during a 1-month trial period of bracing may improve the cost-effectiveness of bracing and eliminate a large percentage of treatment failures.

Complications of brace treatment have not been previously described. Among the 46 patients reviewed, 1 patient had severe ipsilateral leg swelling and symptomatic pulmonary embolus, diagnosed 10 days after brace issue and confirmed by a ventilation-perfusion scan. Another patient discontinued brace use within 2 weeks after experiencing severe ipsilateral leg swelling that was not further investigated. These two patients were the oldest patients in this series at ages 82 and 84 when the brace was issued and had comorbid factors that made them poor surgical candidates. The patient with the pulmonary embolus had age, limited mobility, coronary artery disease, and a history of cerebrovascular accident as risk factors for deep-vein thrombosis. The patient with unilateral leg swelling had age, obesity (BMI of 31), and limited mobility as risk factors for deep-vein thrombosis.

Conceivably, tight bracing of the knee could cause venous stasis in the limb and lead to deep-vein thrombosis and pulmonary embolus. Based on the results of this study, however, one cannot say with certainty that there was a direct causal relationship between bracing and the pulmonary embolus in this patient, though the timing is suggestive. When thinking about brace treatment for a patient with risk factors for deep-vein thrombosis or for the elderly patient, one may be prudent to proceed with caution. One also may be wise to counsel patients regarding these potentially serious complications and to have a high level of suspicion for thrombosis or a thromboembolic event in a symptomatic patient with recent initiation of brace use.

This retrospective study does have limitations. Because all braces issued in the specified time period were reviewed, the patients had varying severity of osteoarthritis and varying activity levels. In addition, knee society scores at the initiation of bracing were not reliably done, so nothing can be said about progression of pain or deterioration of function over the entire bracing period. Finally, one should be aware that this study was conducted entirely in a managed-care setting (a Department of Veterans Affairs hospital). Bracing results may be different in a fee-for-service setting.

\section{CONCLUSIONS}

Despite these limitations, several important observations and conclusions can be drawn from this study. Load-shifting brace use for osteoarthritis involving primarily one compartment of the knee was found to be successful in a majority of patients 3 years after brace issue, with patients reporting some benefit, primarily with pain. A large proportion of the failures occur early in treatment, raising the possibility of intervention to eliminate these failures. Though load-shifting brace use seems to be a treatment modality with minimal risk, one should be aware of the potential complication of venous thrombosis and thromboembolism. 


\section{ACKNOWLEDGMENTS}

I thank Philip Lavori, $\mathrm{PhD}$, for his help in the statistical analysis of this manuscript. I also thank Emilia GreenRiviere, RNP, and George Bennett for their contributions.

\section{REFERENCES}

1. Draper ERC, Cable JM, Sanchez-Ballester J, Hunt N, Robinson JR, Strachan RK. Improvement in function after valgus bracing of the knee. J Bone Joint Surg Br 2000;82B: 1001-5.

2. Lindenfeld TN, Hewett TE, Andriacchi TP. Joint loading with valgus bracing in patients with varus gonarthrosis. Clin Orthop 1997;344:290-97.

3. Kirkley A, Webster-Bogaert S, Litchfield R, Amendola A, MacDonald S, McCalden R, Fowler P. The effect of bracing on varus gonarthrosis. J Bone Joint Surg Am 1999;81A: 539-48.

4. Komistek RD, Dennis DA, Northcut EJ, Wood A, Parker AW, Traina SM. An in vivo analysis of the effectiveness of the osteoarthritic knee brace during heel-strike of gait. J Arthroplasty 1999;14:728-42.

5. Matsuno H, Kadowaki KM, Tsuji H. Generation II knee bracing for severe medial compartment osteoarthritis of the knee. Arch Phys Med Rehabil 1997;78:745-49.

6. Hewett TE, Noyes FR, Barber-Westin SD, Heckman TP. Decrease in knee joint pain and increase in function in patients with medial compartment arthrosis: A prospective analysis of valgus bracing. Orthopedics 1998;21:131-38.

7. Barnes CL, Cawley PW, Hederman B. Effect of CounterForce $^{\mathrm{TM}}$ brace on symptomatic relief in a group of patients with symptomatic unicompartmental osteoarthritis: A prospective 2-year investigation. Am J Orthop 2002;31(7): 396-401.

8. Insall JN, Dorr LD, Scott RD, Scott WN. Rationale of the Knee Society clinical rating system. Clin Orthop 1989;248: 13-14.

9. Kellgren JH, Lawrence JS. Radiological assessment of osteoarthritis. Ann Rheum Dis 1957;16:494-502.

Submitted for publication January 6, 2003. Accepted in revised form September 6, 2003. 